\title{
Algunas observaciones \\ sobre la influencia africana en el \\ mosaico hispanorromano
}

JOSE MARIA ALVAREZ MARTINEZ

Un tema bien extendido en los estudios de la musivaria occidental ha sido el de determinar el grado de influencia que los talleres y escuelas norteafricanas desarrollaron en otras regiones más o menos limítrofes como la Galia, Hispania y la propia Península Itálica y sus islas.

Estas consideraciones han tenido como base la espléndida realidad de las escuelas que se desarrollaron en el Norte de Africa, a partir de finales del siglo I d. C., y sobre todo en el período comprendido entre mediados del siglo II y los finales del siglo III d. C., el que se ha dado en llamar "la Edad de Oro del mosaico norteafricano" (1). Esas destacadas series musivas que han proporcionado conocidos yacimientos que se alinean de Tripolitania a la Tingitana,y que muestran un $\sin$ fin de bellos motivos ornamentales, entre ellos las notables producciones del denominado "estilo florido", de conocidas representaciones mitológicas, o de asuntos de la vida cotidiana, son las que han hecho pensar a diversos autores en una cierta dependencia de las mismas de algunas escuelas que se desarrollaron en la Galia, Italia o la Península Ibérica y que, indudablemente, ofrecen temas y representaciones similares y, por qué no decirlo, en ocasiones, estilos muy cercanos. 
Desde luego, el gran número de pavimentos recuperados en esa prolífica región, que se vió favorecida por la administración romana en el período referido, permite a los especialistas realizar estudios que, de día en día, van determinando la personalidad y la variedad de las escuelas que surgieron en ese área geográfica (2).

Dunbabin opinaba (3) que los primeros contactos entre los mosaistas hispanos y los norteafricanos pudieron haber tenido lugar a comienzos del siglo III d. C. Con posterioridad, las relaciones se estrecharían como pone de manifiesto la repetición de las representaciones, similares en uno y otro caso, bien por esos contactos directos aludidos o por el uso de los mismos modelos. Llega a pensar la distinguida profesora que el auge en la realización de mosaicos hispanos en el siglo IV d. C. pudo motivar, incluso, la presencia de musivarios norteafricanos en busca de trabajo, que influirían en la temática y en el tipo de composiciones muy similares en muchos casos a las norteafricanas. Esta fue, igualmente, nuestra reflexión a propósito del estudio de los caracteres de los mosaicos de la villa de "El Hinojal", donde apreciábamos reflejos africanos, más concretamente de la zona de Cartago (4).

Esta situación era también apreciable en otras regiones como Italia, y, sobre todo, Sicilia. A propósito de los mosaicos de la isla y su relación con los del Norte de Africa, Wilson (5) llegó a lanzar tres hipótesis a manera de explicación del parentesco entre unas y otras producciones:

a) Los mosaicos sicilianos pudieron ser realizados por artesanos africanos.

b) Los talleres africanos pudieron haber tenido filiales en la isla.

c) Se utilizaron los mismos cartones, sin atender a la personalidad de una y otra escuela.

Parecidas consideraciones se hizo Johnston a propósito de la posible influencia de los mosaicos africanos en los británicos: que mosaistas africanos visitaron, o se establecieron en Gran Bretaña; que repertorios o manuscritos originarios del Norte de Africa se usaron en Britannia o, lo que parece más probable, que las influencias llegaron indirectamente, de acuerdo con la difusión de los temas en el Imperio (6).

Por su parte, entre nosotros, el Prof. Blázquez, que ha realizado una meritoria labor de estudio y difusión de los mosaicos hispanos y ha dado 
aliento a un buen equipo de especialistas, ha incidido en numerosas ocasiones en la influencia africana de algunas de nuestras series musivas de los siglos III y IV d. C. fundamentalmente. Sus consideraciones son muy interesantes y la evidencia está presente en muchas de ellas, pero, aceptándola, opina que se ha exagerado un tanto esta influencia y que a veces las similitudes pueden responder al empleo de un modelo común (7).

Otros estudiosos han minimizado esta influencia africana en nuestras producciones. Tal es el caso de Fernández-Galiano (8), quien ha llegado a cuestionar la dimensión de esa posible dependencia.

En verdad, el panorama dista mucho de ser claro. Es cierto que se aprecian influencias, pero no lo es menos que las afinidades afectan a cuestiones de carácter general, fundamentalmente a aspectos iconográficos, mientras que los esquemas compositivos, y los temas ornamentales, que, en ocasiones, pueden definir más a una escuela o variante, no se han analizado con la profundidad que sería de descar, aunque se está en el camino correcto gracias los programas de estudio que se han llevado a cabo en los últimos tiempos tanto en un sitio como en otro. Hoy, en nuestra opinión, no estamos en condiciones de presentar sino una leve aproximación al problema.

Pero esas relaciones y similitudes en las producciones musivas existen.

Es claro, como se ha dicho en numerosas ocasiones, que las relaciones que mantuvo Hispania con el Norte de Africa a lo largo de varios siglos fueron abundantes. Blázquez (9) ha enumerado repetidamente los momentos y las circunstancias en los que esos contactos se hicieron más patentes (10). Estos se incrementaron considerablemente durante el Bajo Imperio y es significativo a este respecto el gran número de testimonios que vinculan a la iglesia hispana, en especial a la emeritense, con la de Cartago, que era regida por la poderosa figura de San Gipriano, quien llegó a enviar una carta a la

atribulada diocesis augustana inmersa en una crisis por la apostasía de su metropolitano (11).

Existieron, además, a lo que parece, abundantes contactos comerciales entre ambas regiones y en el caso, también, de la colonia Augusta Emerita, 
la importación de la denominada sigillata africana fue una práctica común ya desde finales del siglo I d. C. y la segunda centuria, fecha en la que llegan las correspondientes al tipo $A$ procedente de los alfares del Norte de Túnez, aunque con escaso número de ejemplares y fruto de un comercio todavía ocasional, para generalizarse ya en la segunda mitad del siglo II d. C. y primera mitad del siglo III d. C..La verdadera invasión de ejemplares africanos, correspondientes ya a la variedad $C$, realizados igualmente en la Bizacena, se produce a partir de la primera mitad del siglo III y su mayor presencia en el mercado emeritense se puede situar entre el 240 y el 350 , aunque en la cuarta centuria son ya piezas correspondientes a la forma $D$ las que lo ocupan hasta la primera mitad del siglo $V$ d. C. (12)

RELACIONES ENTRE LAS ESCUELAS NORTEAFRICANAS E IISPANAS.

TIPOS ICONOGRAFICOS

Estas relaciones se han querido apreciar en una serie de motivos iconográficos que corresponden a conocidas series, y que se repiten en ambas regiones. Son, fundamentalmente, asuntos báquicos, temas nilóticos, escenas de circo y de la vida cotidiana, en especial los lances cinegéticos, entre otros varios.

\section{ThMas baQlicos}

Los temas del ciclo de Baco, en particular aquellos que contienen una representación de su cortejo poblado por ménades, sátiros, faunos y personajes bien conocidos como Pan y el viejo Sileno, o los que presentan el propio triunfo del dios, son bien frecuentes en la musivaria romana, en varias épocas que podemos situar, en los momentos de mayor proliferación de escenas, entre el siglo II d. C. y el siglo IV d. C., sin que falten algunos ejemplos posteriores a esa cronología. Los tipos iconográficos se acunaron bien pronto (13), en época helenística y se repitieron hasta la saciedad no sólo ya en mosaicos, sino también en pinturas, relieves, fundamentalmente en sarcófagos, y en ciertas artes menores. 
Las escuelas norteafricanas igualmente prestaron importancia a estos temas, pero quizá hubo un interés más marcado por la representación del Triunfo de Dionisos. Así (14), desde finales del siglo II, o comienzos del siglo III d. C. aparece una larga serie con este tipo de escenas: Hadrumetum, El Djem, Cherchel, Tipasa, Sabratha, Saint Leu etc. Todas ellas tienen una caracteristica común y es la uniformidad, a pesar de las variantes cronológicas y de detalle (15). Dunbabin ya explicaba esa uniformidad por la dependencia de todas ellas de un modelo común, que se interpretaría de una u otra manera en las diferentes composiciones (16).

Por su parte, en Hispania surgieron otras representaciones del Triunfo de Baco en mosaicos de Zaragoza, Ecija, Itálica, Cabra, Torre de Palma, Baños de Valdearados, Liédena,Tarragona, ejemplares bien conocidos, con una cronología que va de la mitad del siglo II $\mathrm{d}$. C. hasta el siglo IV $\mathrm{d}$. C. (17), a los que habría que añadir el más recientemente aparecido en la provincia de Badajoz, en la Finca "Torre Albarragena", cerca de San Vicente de Alcántara (18).

A partir de esos abundantes ejemplos que proporcionan tanto las escuelas norteafricanas como las hispanas se han querido establecer relaciones entre ambos grupos, basadas en las coincidencias iconográficas de varios de estos pavimentos. Así, Blanco ya observó justamente afinidades entre el Mosaico de Zaragoza y los de Hadrumetum y El Djem (19).

Por su parte, Blázquez (20) también apreció estas similitudes bien evidentes, en cuanto a aspectos iconográficos y a tipos compositivos. El Mosaico de Zaragoza (figura 1) (21) habría que relacionarlo con varios ejemplares norteafricanos: con los de Hadrumetum (figura 2), El Djem y Cherchel (22) por la forma de carro que aparece en ellos, muy similar. Igualmente, la consideración 


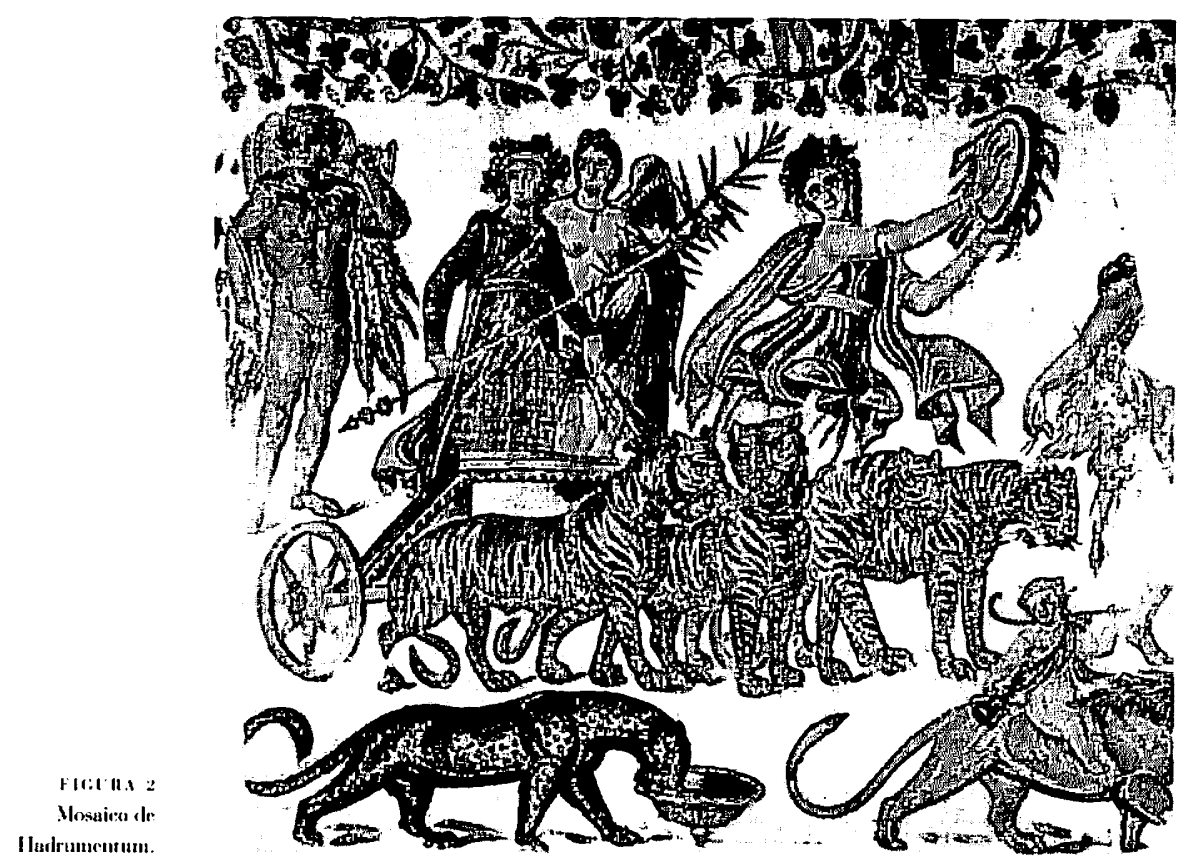

de la figura del dios sería muy semejante en los mosaicos de Zaragoza y El Djem. Por fin, la figura de Victoria que aparece coronando al dios en Zaragoza es muy cercana a las que se aprecían en Hadrumetum y El Djem. Otros mosaicos hispanos reflejarían rasgos comunes con sus congéneres africanos. Así, el de Ecija (23), de fines del ll d. C. se emparentaría con el de Hadrumetum; el de Itálica (24), de fines del II o comienzos del III d. C., con los de El Djem y Sabratha; el de Cabra (25), de la primera mitad del siglo III d. C., con los de Thysdrus y Saint Leu. Finalmente, los más tardíos de Torre de Palma (26) y Liédena (27) con los de Thysdrus y Hadrumetum respectivamente.

Las relaciones y similitudes entre ambas series son innegables: existen afinidades iconográficas: se refleja el mismo esquema compositivo; es significativa la presencia de figuras no tan frecuentes como la Victoria entre varios ejemplares. Está muy claro todo ello; pero, ahí está la pregunta: ¿Quién influye en quién?. ¿El mosaico de Hadrumentum, cabeza de la serie norteafricana, que, segun la autorizada opinión de Dunbabin, dataría del 200-210, sería el modelo en el que se fijó el musivario cesaraugustano, que realizó su obra en 
la segunda mitad del siglo II d. C.? No parece probable; o al menos resulta difícil admitirlo.

Aun aceptando esas relaciones, que, repetimos, son evidentes, entre los mosaicos norteafricanos y los hispanos, hay que convenir que el modelo, el tipo iconográfico, es mucho más antiguo que los ejemplares africanos e hispanos; se había acuñado mucho antes, en el mundo helenístico, y se repetía en un sin fin de ejemplos, - fundamentalmente relieves sarcofágicos-, algunos de los cuales resultan ser nuestros mosaicos.

Es muy probable que tanto en una región como en otra los musivarios utilizaran un modelo común, al que se aplicaron pocas variantes de detalle en uno y otro lugar, aunque habría que determinarlas mejor para reflejar la verdadera personalidad de ambos grupos, aun por definir en nuestra opinión y, en consecuencia, apreciar las posibles relaciones e influencias entre las escuelas, si es que la hubo y no evolucionaron independientemente como llega a pensar. quizá correctamente, el Dr. Fernández-Galiano (28).

Pensamos, en definitiva, a falta de un estudio más ajustado, que está por hacer, en una interdependencia, en una dependencia de un modelo común de las escuelas de ambas regiones.

Lo mismo podemos decir de otras representaciones del ciclo báquico, bien abundantes. Blázquez (29) refiere esa representación del dios unida a las Estaciones, que vemos, por ejemplo, en Itálica (30), parangonable con varios ejemplos africanos, entre éllos uno de Volúbilis, pero sin que podamos, en modo alguno establecer una dependencia hispana de los ejemplos africanos, y, sí, como en el caso anterior de la serie de pavimentos del Triunfo de Dionisos, considerar la repetición de un modelo común. 


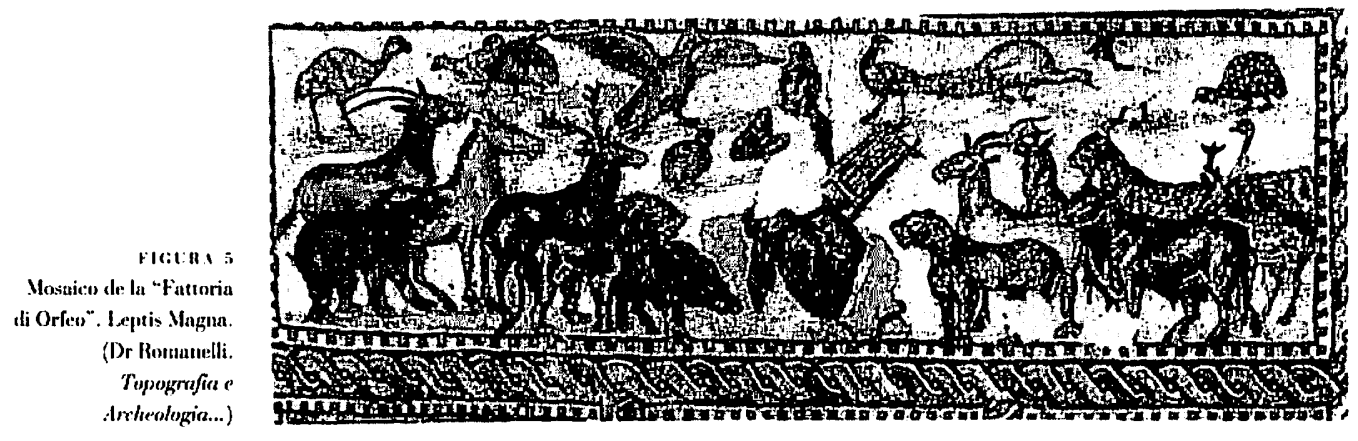

Sería el mismo caso que otras representaciones de carácter dionisíaco como las que muestran al viejo Sileno, ebrio, sobre un pollino, tema iconográfico definido de antiguo y que aparece en varios pavimentos hispanos, entre éllos los de Mérida (figura 3) (31), Conimbriga (32) y Liédena (33), y en alguno africano como el de la Chebba (figura 4).

\section{IAS REPRESENTACIONES DE: ORIFO}

No ofrece el Norte de Africa la riqueza de representaciones de Orfeo, en particular en su episodio de cantar ante los animales, hallada en Hispania, donde se reflejan todos los tipos iconográficos sistematizados en su día por Stern (34). Sí se consideró, en su momento, la escena que contiene el Mosaico hallado en la "Fattoria di Orfeo" de Leptis Magna (35) como prototipo del tipo II (figura 5), que se extendió por diversas regiones del Imperio, entre éllas Hispania (36). El esquema, segun las opiniones más recientes, pudo tener su origen en composiciones relivarias.

En el Norte de Africa, concretamente en Volubilis (37), apareció otro pavimento, esta vez correspondiente al tipo III, hasta entonces privativo de las Islas Británicas (38), y al que hay que sumar ya un ejemplo emeritense (39).

RSCEXAS NILOTICAS

La egiptomanía en el arte romano es evidente en algunos períodos del Imperio. Es importante el impacto que produjo la conquista de Egipto en la sociedad romana, a la raya del Imperio. Su conocimiento supuso una 
difusión de los temas egipcios (40), y, sobre todo, de aquellos con una cierta vis cómica acuñados en el entorno alejandrino.

La serie de mosaicos nilóticos africanos, bien conocida en sus representaciones por el estudio, entre otros de Foucher (41), ofrece una amplia variedad y riqueza. Los pavimentos hispanos aparecidos hasta el momento son casi tan importantes como aquellos, aunque bien hay que decir que estas representaciones hispanas no adoptan un protagonismo claro dentro del pavimento, sino que forman parte de la composición como un motivo de adorno o de relleno más que otra cosa.

Tal es el caso de los mosaicos italicenses de la "Casa de Neptuno" y de la "Casa de la Exedra" (42), o el de los emeritenses de la Casa de la calle Sagasta (43), y de la hallada en la Travesía de Pedro María Plano (44), con escenas un tanto convencionales, con figuración de paisaje levemente sugerido por elementos de la flora nilótica, más completo el de la calle Sagasta, y con escenas nuevas en el caso del también emeritense de la Travesía de Pedro María Plano. Más interés muestra el mosaico de Puente Genil, donde aparece una escena cómica como centro de la composición, en modo alguno accesoria, cuyos protagonistas son pigmeos (45̃)

Estas escenas no tienen, como las anteriormente referidas, otra relación que la que motiva su origen común, muy antiguo y bien presente en los repertorios de la pintura pompeyana (46), donde aparecen acuñados perfectamente los tipos iconográficos.

EL, MUNDO DE IOOS ESPlECTACLLOS

Otras escenas relacionadas con el mundo de los espectáculos también han provocado comentarios igualmente sobre la dependencia de los mosaicos hispanos de los norteafricanos.

Las escenas de palestra son frecuentes en el mundo romano. En Hispania no han sido muy abundantes, al igual que en Africa. Dependen de modelos antiguos bien determinados.

Más interés ofrecen los temas circenses, abundantes tanto en Africa como en Hispania, sobre todo la representación de aurigas vencedores, aunque igualmente habría que considerar alguna vista del momento de celebración de los juegos. 
En este caso habría que citar dos excelentes mosaicos hispanos, donde aparecen los carros dando vueltas alrededor de la spina: el de Bel-Lloch y el de Barcelona (47), que se pueden parangonar, por su importancia, con el de Cartago (48).

Pero donde podemos apreciar más relaciones es en la serie, antes referida, de los aurigas vencedores, a veces identificados con sus nombres, al igual que algunos de los caballos del tiro que conducen. El tema ha sido estudiado perfectamente por Dunbabin, quien habla de la aparición de estas representaciones a partir del siglo III como una constante y sugiere ciertas variantes de detalle (49). El auriga suele aparecer en posición frontal, sobre la cuadriga, con la fusta en la mano derecha y con la palma del triunfo en la izquierda, $o$, a veces, con la corona en la derecha y la palma en la izquierda, tal y como lo vemos en Mérida (figura 6), incluso en una pintura del siglo IV d. C. (50).

Esta serie hispana tiene sus evidentes relaciones con las africanas, porque obedecen a un modelo común, bien estereotipado. No podríamos

FIC, RA "

Mlasaicu con representumión del

auriga, Mforriumus. Móriıla.

Mluseo Nacionnl de Arte llomano.

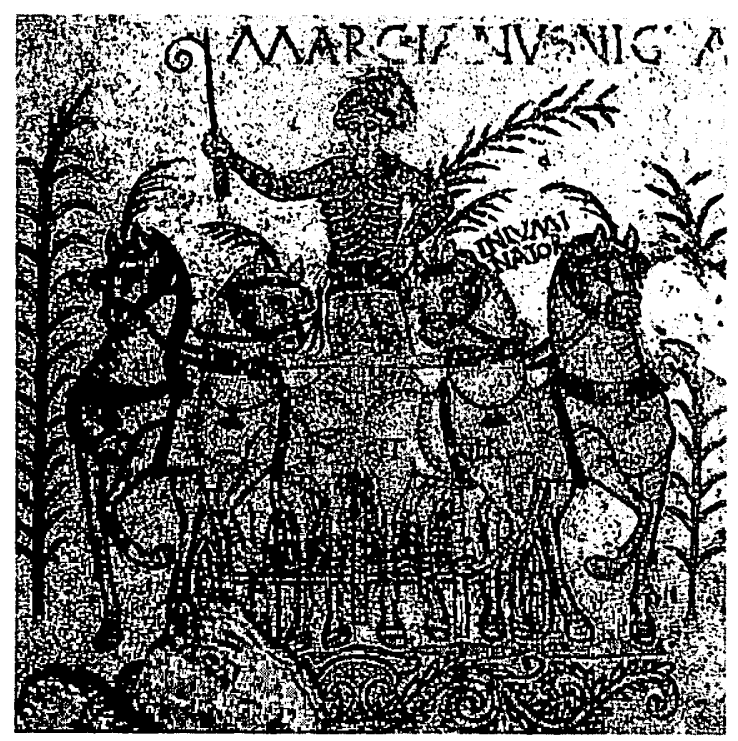
silenciar el caso del auriga Quiriacus, muy semejante en su esquema a los hispanos, pero no estamos legitimados para considerar que los cartones hispanos dependan de los de Africa Proconsular, por estas similitudes del tipo iconográfico, ya establecido de antiguo. Sí, en cambio.,es interesante observar cómo hay una relación evidente entre los temas dionisíacos y el mundo de los ludi, tanto en Africa como en Hispania. Valgan los ejemplos de El Djem, donde, en la Casa de Baco, aparece Dionisos entre las fieras del anfiteatro, y el de Mérida, donde. en el pavimento hallado en la calle 
Arzobispo Massona, se ven escenas báquicas entre los dos cuadros de los aurigas.

\section{LOS ASINTOS DE LA VIDA COTIDIANA}

Es en esta serie, más importante en Africa por el gran número de representaciones conservadas, y por su variedad, donde hallamos modelos y esquemas iconográficos que sí tienen una relación más marcada con nuestras producciones musivas. Gracias a las representaciones de la vida rural que ofrecen los pavimentos africanos, se pueden reconstruir pormenores de aquella sociedad de los siglos III y IV que desarrolló una existencia llena de esplendor presidida por el buen hacer en los campos (51).

La variedad de representaciones de villae en los mosaicos norteafricanos, bien sistematizada por Sarnowski (52), nos permite, además de conocer la estructura de estos establecimientos, hacer una comparación con los ejemplares hispanos, no tan abundantes (53).

Una representación similar, bien analizada en su relación con los ejemplos africanos, es la que aparece en las diversas escenas del conocido mosaico de Arróniz (figura 7) (54). En el se aprecían vistas de una villa, que podría encontrar elementos similares en varios mosaicos, entre los que podemos citar los de Cartago, Henchir Toungar, Oudna o Tabarka (figura 8) (55). Son imágenes, además, muy cercanas en el tiempo: fines del siglo IIl o comienzos del IV d. C. Es lo que podemos decir, igualmente, de otros ejemplos bien conocidos como los de Centcelles (56).

No hay en Hispania un mosaico tan completo como el del Dominus Iulius de Cartago (figura 9) (57), donde se refleje el ciclo de las Estaciones con frutos alusivos a

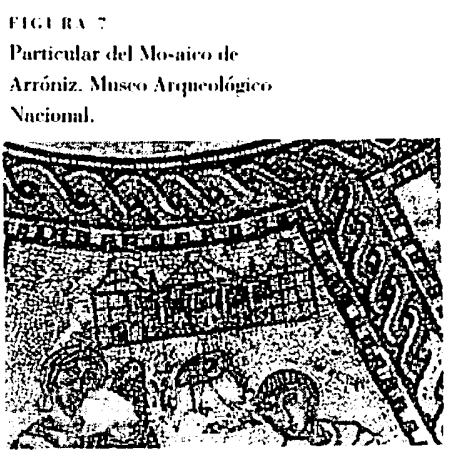

116,1818

Mlosuico de Tinharkia.

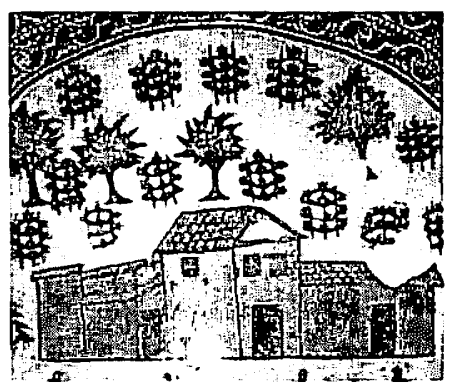

II:I R.1"

lominus Iulins.

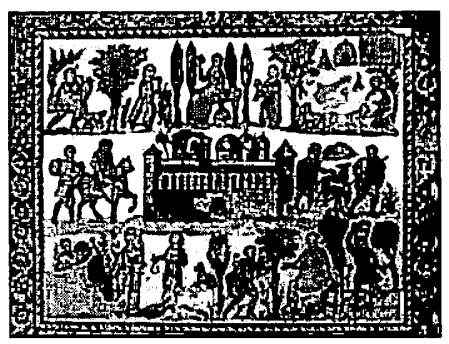

IItens 10

Mosaico de -Panes Perdidos".

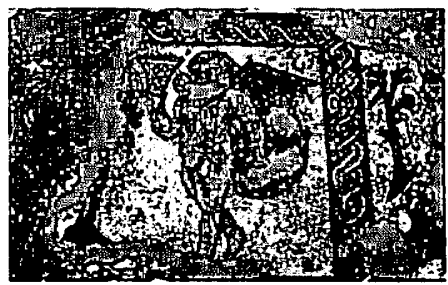


FI1:1114 11

Particulur del mosuico de

la vemlinia. Casa del

Anfiteatro. Mérida.

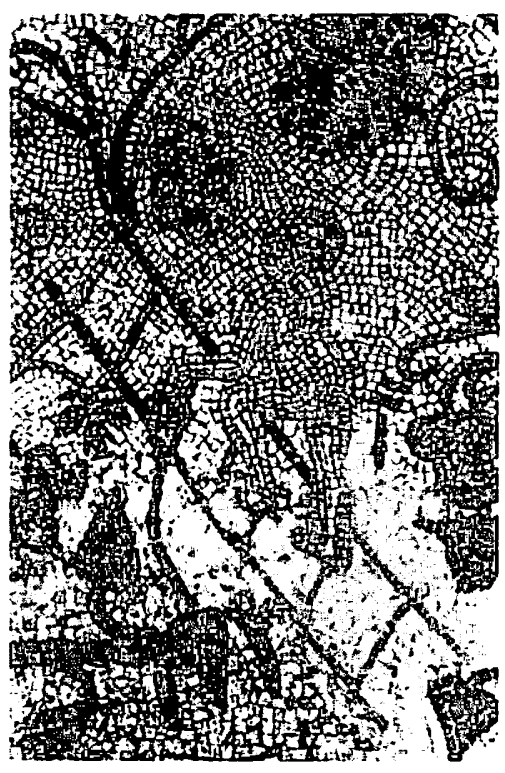

las mismas, pero de ese espíritu participan algunos mosaicos hispanos como el de "Panes Perdidos" en Solana de los Barros, muy destruido, pero con clara alusión a la felicitas temporum que preside la vida rural. En el figuraban escenas alegóricas relacionadas con las estaciones y sus frutos (figura 10) (58).

Entre las tradicionales labores que ofrece la representación de la vida rural, destaca, por su presencia en diversos pavimentos itálicos, hispanos y africanos, el ciclo de la vendimia practicada bien por putti o por campesinos a los que se sorprende en el momento de recoger los racimos de las viñas, o de las altas parras, de transportar la uva hacia el lagar, o en la acción de la pisa del fruto. Estos mosaicos son bien conocidos y podríamos citar el de la "Casa del Anfiteatro de Mérida”, uno de los más emblemáticos, donde se aprecian diversos erotes subidos a las parras (figura 11) y a tres operarios enlazados por sus manos en el momento de proceder a la pisa de la uva (59). Algo parecido, esta vez sin la escena de las labores de aludidas, pero con la presencia de un carretero que conduce el fruto al lagar, se observa en otro mosaico emeritense de la calle Travesía de Pedro María Plano (60). No faltan estas escenas en otros pavimentos hispanos como los de Sagunto, con érotes vendimiadores (61), Calpe (62) y Complutum (63).

Estas representaciones suelen ser frecuentes en mosaico a partir del siglo III, aunque existen ejemplos anteriores. Probablemente, derivan de composiciones pictóricas helenísticas. Los mosaicos son muy numerosos en el Norte de Africa, donde aparecen antes que en la Península Ibérica. Destacaríamos, entre otros, dos muy característicos: los de Cherchel (figura 12) y El Jem (64).

Entre las escenas hispanas y las norteafricanas existe un evidente paralelismo como muestran tanto el tratamiento de las parras, que suelen originarse en kantharoi, y la presencia de campesinos vendimiadores y érotes alados provistos de cestos o cubos, de escaleras y de instrumentos típicos de la recolección. 
No podemos silenciar a este repecto el mosaico de la cúpula del Mausoleo de Santa Constanza, donde aparecen los mismos vendimiadores, el carro cargado de uva y la escena de la pisa del lagar (65).

Quizá donde se establecen más relaciones, si no nos parecen suficientes los ejemplos ya referidos, entre los mosaicos hispanos y los norteafricanos es en las escenas de caza, bien numerosas en uno y otro lugar.

La afición de la caza por los hispanos está presente en las fuentes clásicas (66) y todo ello se tradujo en un sin fin de representaciones con diversos lances cinegéticos, ora en episodios aislados, ora en escenas abigarradas en las que participan varios cazadores. Esos lances comprenden todo un muestrario del arte de la caza: caza a caballo, a pie, con ayuda de perros, con redes etc.

En una de las más considerables villae del entorno de la colonia Augusta Emerita, la de "El Hinojal" en la dehesa de "Las Tiendas", aparecieron dos escenas cinegéticas de interés. En la primera de éllas, en un mosaico que se situó, a la vista de todos, en un posible oecus con función de triclinium, se representa a un cazador, probablemente el dueño del fundus que alancea con valor, siguiendo al punto las prescripciones de Jenofonte en su "Arte de la caza", a un jabalí con el que ha mantenido un feroz cuerpo a cuerpo, en medio de un paisaje de monte bajo, de dehesa extremeña (figura

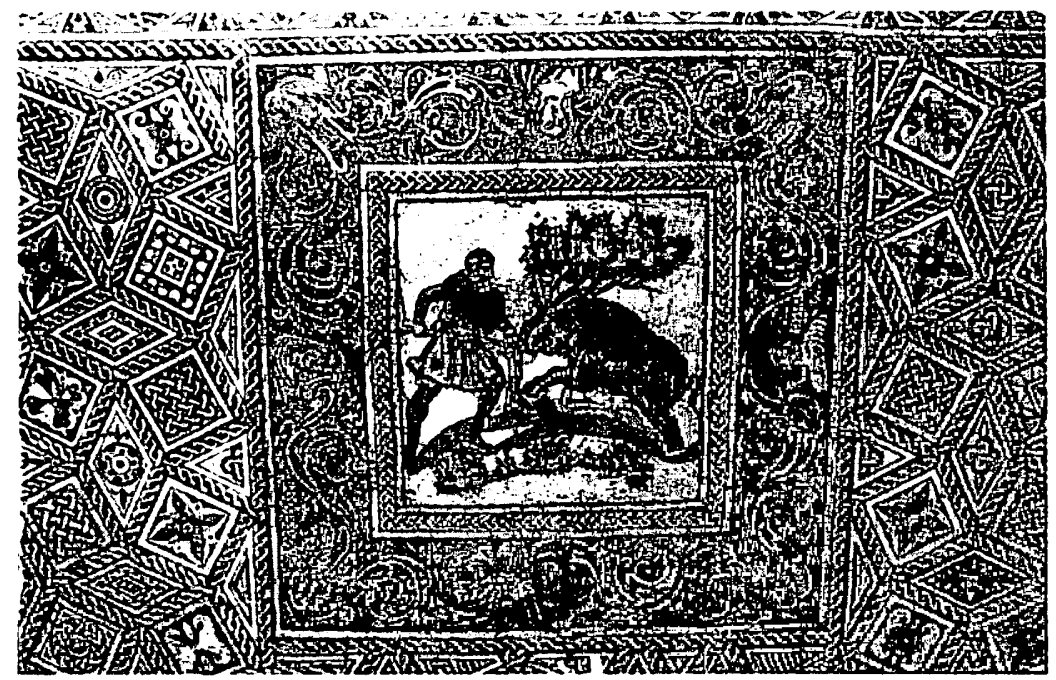


13). También, en una suerte de paradeisos, la irreflexiva figura de un caballero aparece en el momento de alancear a un felino al que ha dado alcance tras una veloz carrera (67).

El tema con su carga simbólica como exaltación de la virtus de aquella sociedad hizo fortuna y se repitió en otros pavimentos del entorno de la colonia augustana, como el de la villa de "Panes Perdidos", donde aparecen escenas cinegéticas con su protagonista y su caballo, en un esquema muy próximo al del conocido mosaico del Antiquarium de Cartago (68), y al de

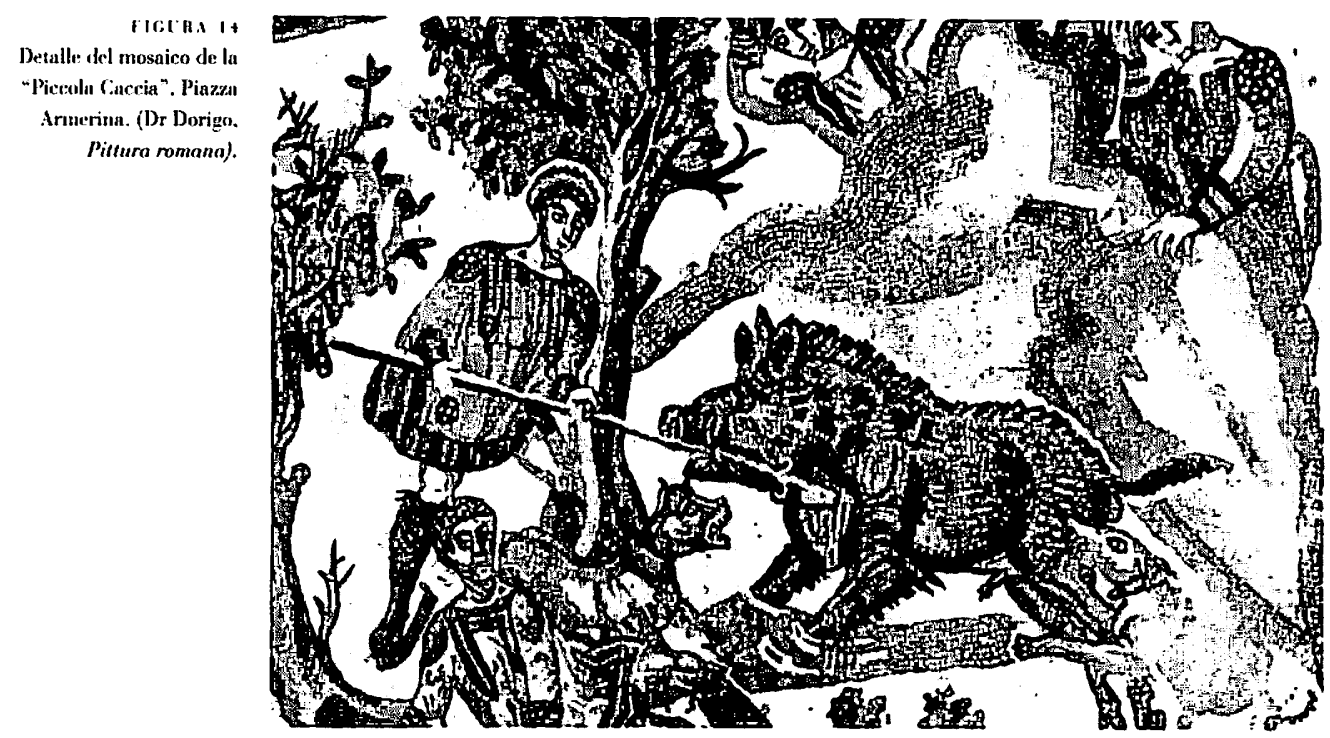

otro mosaico emeritense, en verdad excepcional, el hallado en la calle Holguín, en el que se ve a un cazador, de nombre Marianus, sin duda el dueño de la casa, con su caballo Pafius, orgulloso con su trofeo, un ciervo (69).

Estos mosaicos emeritenses, que nosotros adscribimos a una misma escuela (70), muestran en su composición los caracteres que observamos en otros congéneres norteafricanos, sobre todo los producidos en la zona de Cartago, y responden a los cánones de las representaciones del siglo IV d. C. En lo que atañe a los paralelos del Norte de Africa, podríamos referir el de Cartago con escena semejante a uno de los cuadros del mosaico de la calle Holguín (71), u 
otros parecidos de Djemila y Cherchel donde se observan sendos cazadores a caballo en el momento de arrojar sus jabalinas, tal y como sucede en el mosaico emeritense (72), Oudna (73), Museo Bône (74) etc.

Por otra parte, una escena similar a la de la cacería del jabalí del mosaico de "El Hinojal", aparece en Piazza Armerina (figura 14) (75).

Otros ejemplos hispanos podrían ser referidos en este parentesco con composicionmes norteafricanas. El recuerdo del mosaico de Dulcitius, de la villa romana de El Ramalete, es fácil de evocar, puesto que presenta una composicion muy similar. El motivo, bien estudiado por Blázquez y Mezquiriz (76), ofrece numerosos paralelos, además de con el mosaico emeritense del cazador a caballo de la calle Holguín,con pavimentos norteafricanos con los que se emparenta próximamente: El Djem, Cartago, Hippo Regius, Bord-Djedid (77). En este caso mucho se ha discutido sobre el estilo del pavimento y sus posibles influencias oriental o africana, destacándose, en todo caso, su "facies" occidental.

Otro mosaico que ofrece cierto parentesco con las representaciones cinegéticas africanas en friso corrido, o sucesión de escenas, es el de Centcelles.

Es preciso referir que otros pavimentos cinegéticos como el aludido de la villa de "El Hinojal", con representación de un caballero persiguiendo a una pantera, o el de la villa de "La Olmeda", a nuestro juicio, responden ya a rasgos de la corriente oriental, bien perceptible en la Península a partir del s. III d. C. y caracterizada por el gusto por lo pictórico, la espacialidad, la búsqueda de efectos volumétricos, el tratamiento ilusionista y ese característico "galope volante", típico de las composiciones de Antioquía (78).

Una vez que hemos analizado la problemática que atañe a la influencia africana en nuestras producciones musivas, podemos percatarnos de lo lejos que aun estamos de determinar cuál fue el grado de esta posible influencia y cuál, igualmente, el de la nuestra en la zona norteafricana, pues estamos convencidos que durante el Bajo Imperio, al menos, se produjo esa influencia mutua en regiones tan próximas como las nuestras (79).

Hasta el momento no podemos hablar de otra cosa que de relaciones mutuas, de una interdependencia en los modelos iconográficos de un arquetipo comun. En todo caso, en una y otra región, la influencia itálica es evidente. 
I G. Ch. Pieard. "L'âge d'or de la mosaïque romaine en Afrique du Nord". Les dossiers de l'Archéologie, n" 31 (novembre-décembre 1978), pp. 12 ss.

2 Un magnífico estudio de síntesis sobre las producciones norteafricanas es el de K. $\mathrm{Y}$. D. Dunbabin. The Mosaics of Roman North Africa. Studies in Iconography and Patronage. Oxford Monographs on Classical Archaeology. Oxford, 1978.La obra de la Prof. Durbabin vino a sistematizar un buen número de cuestiones planteadas en trabajos anteriores. Un ejemplo reciente de lo que supone el estudio ajustado de la producción musiva en una región y de su área de influencia ofr:: S. Gozlan. Ia Maison du Triomphe de Neptune à Acholla (Botria, Tunisie). I. Les mosaïques Collection de l'Ecole Française de Roma-160. Roma, 1992. Hay que destacar, de igual modo, el esfuerzo llevado a cabo sobre los mosaicos de Túnez por el Instituto Nacional de Arqueología y Arte de Túnez en colaboración con un grupo de instituciones americanas, que ha dado como resultado la publicación de varios fascículos del Corpus des mosaïques de Tunisie, bajo la dirección de M. Alexander y M. Ennaifer.

3 K. M. D. Dunbabin, op. cit., pp. 219-220.

4 J. M. Alvarez Martínez. "La villa romana de "El Hinojal" en la dehesa de "Las Tiendas"(Mérida). N.A.H. -Arqueología4 (1976). Decíamos en ese estudio (p. 458): "Creemos que se trata de artistas conocedores de la corriente africana que triunfa plenamente en occidente desde finales del siglo III d. C. y que tuvo ramificaciones claras en Sicilia, Galia, Italia etc. No hay que desechar de ningun modo la hipótesis de que los artífices de nuestros mosaicos fueran artistas ambulantes muy relacionados o procedentes de una zona próxima a Cartago que trabajaron para unos clientes que les imponen su gusto...”.
5 R. J. A. Wilson. "Roman mosaics in Sicily; The African connection". A.J.A. 86, 1982, pp. 413-428, y sobre 1odo pp. $425 \mathrm{ss}$. Id. "Mosaics, mosaicists and patrons". JRS, 71, 1981, pp. 173-177. En las recensiones de varias obras, entre cllas la de Dunbabin, se consideran las razones, que luego desarrollaría ell el artículo de A.J.A.

6 D. E. Johnston. "Some possible Nordh African influences in Romano-British mosaics". Fith International Colloquium on Ancient Mosaics. (Bath, 1987), Amn Arbor, 1994. pp. 295 ss. y resumen en p. 304.

7 Varios son los estudios en los que el Prof. Blázquez ha hablado de las relaciones de nuesiras producciones musivas con las del Vorte de Africa. Valga como muestra uno de sus más interesantes trabajos: J. M.

Blázquez-G. López Monteagudo-M. P. García Gelabert-M. L. Neira "Influjos africanos en los mosaicos hispanos". L'Africa romana. Atti del VII Convegno di studio. Sassari, 15-17 dicembre 1989, pp. 673-694.

8 D. Fernández-Galiano."El triunfo de Dioniso en mosaicos hispanorromanos". AEspA, 57, n" 149-150 (1984), pp. 111 ss.

9 J. M. Blázquez et alii, art. cit., pp. 673-74.

10 Una buena síntesis del problema se puede ver en su estudio: "Relaciones entre Hispania y Africa desde los tiempos de Alejandro Magno hasta la llegada de los árabes" en F. Altheim-R. Stiehl, Die Araber in der Alten Well. Berlín, 1969, V. 2 pp. 470-498.

11 Véase sobre este asunto: J. M. Blázquez. "La carta 67 de Cipriano y el origen africano del cristianismo español". Homenaje a Pedro Sainz Rodriguez. III. Estudios Históricos. Madrid, 1986. pp. 93-102. Más reciente es el estudio de R. Teja, quies llega a identificar como obispo emeritense a Basílides y no a Marcial, como hasta ahora se venía admitiendo cfr:: "Mérida cristiana en el siglo 
III: sus primeros obispos" en Merrida y Samma

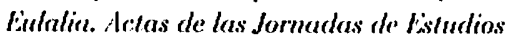
Lisfralicuses. Mérida, 1995, pp. 333-44. si parece muy evidente la dependencia de los modelos africanos en los mosaicos hispanos de temática cristiana, doncle no deja de apreciarse la corriente itálica de los centros del Adriálico fundamentalmente. Solse los mosiaies af ricanos del periodo cyue lausos influyeron en Hispania: N. Duvul. La

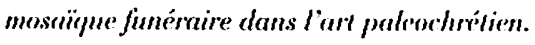
Ravenua, 1976: K. M. Dunhabin "Mosates of the Byzantine period: problems annl directions of research". Cahicess des cifudes anciennes, 18, pp. 9 ss.: M. Eunnafer. "la mosaïyue africaine à la fin de limnicyuicé et au début de l'epoque médievale". Fïh International Colloquium on ancient Mosaics (Bath. 1992), Ann Arbor, 1994. pp. 3017 ss. V'́ase igualmente: J. M. Blázquez Martínez. "Mosatico paleocristiano del Musieo de. Huesen". La romanització del Pirineu. Puigcerdá. 1990, pp. 137-141.

12 A. Vizupuez de la Cueva.Sigillata africano en Augusta Emerita. Monografias limerilenses 3. Mérida, 1985, pp. 89 ss.

13 C. Catsparri, s. e. Dyonisos en LIMC' III. 1. Iri. 496 ss.

14 K. Y. Dunbabin. "The triumph of Dimisos on mosaics in . Vorth Africa".P.B.S.R.. 39 (1971). pp. $56 \mathrm{ss}$.

15 L has son más completas que uras. No es lo mismo la alsigarrada composición que aparece en la "Casa de la Prowesión Dionisiaca” de El Djem (Cfr. L. Fincluer.La Maison de la Procession Dionysingure a El Jem. Publications de l'Universile de Tuntis, 1 re. Serie: Archéologie e Histoire vol XI. Paris. 1963) que la representación de. Sabratha, más resumida.

16 K.M. Dunbabin, op. cit. pp. 181-182.

17 D. Fernindez-Galiano. "EL, Triunfo..." plj. $98 \mathrm{ss.}$
18 A. Cinzzále\% Cordero-M. de Alvarado Gomzález-J. Molano Brías. "Mosaicos de la villa romana de Torre Albarragena. Lin nuevo triunfo báquico en la Penímsula Hhérica" At:sp.A, 633 (1990), pp. 317-3330

19 A. Blanno. "Mosaicos antiguos de asumto háçuiю" B.R.A.H. CXXXI (1952). [i]. $27: 3$ ss.

20 J. M. Blízquez of alii, ar1. cil. p). 675-678

21 D. Fernínde-Galiano. Mosarcos rommos del concento cesurangustamo. Zaraguza, 1987. pp. +2-to.

22 K. .. D. Dunbabin, op. cil., pp. 181-182.

23 I. V. Blizupuez. Corpus de mostricos de Espariar. Fass: IV. Mosnicos romanos de Servilla, Cirusuda, Cádiz y Murcia, Madrid. 1982, ॥" 1, pp. 13-19, láms. 1-2, 38-39.

24 A. Blanco lireijeiro. Corpus de mosaicos romenos de España. Fasc. II. Mosaicos romenos de Lálica (I). Madrid. 1978, 11" 19, Pp. $+(0-+1$. liums $+t-t 5$.

25 I. M. Blázunue. Corpus de mosaicos romanos de Lapoña. lase. III. Mosuicos romenos de Córbloba, Jaen y Málaga. Mardricl. 1981. p. 102. lig. 32.

26 li. de Almeida. "Quelques mosiänues rmmaimes de Portugal" CMCR II.. 1975. p. 222. lám. LXXXI.

27 J. M. Blazquez--M. A. Mezquiriz. Corpus de mosairos romanos de España. liase. VII. Mesnicos romanos de Navarro. Madrid. 1985, n" 24, pp. +4-48, láms. 28-29.

28 D. Permúnde\%-Cialiano. op. cir., p. 111-112.

29 .I. .. Blízques et alii, "Influjes..." p. 679.

30 A. Blanco. Mostaicos romanos de licilica, pp. 27-28.

31 J. M. Alvarez Martinez. Mosaicos romanos de Mérida. p. +2.

32 I. M. Bairno Oleiro. Corpus rlos moscicos somanos de Porlugal. Coneentus 
Scallabitanus. 1. Conimbriga. Casa dos Repuxos. Conimbriga, 1992, pp. 98 ss.

33 J. M. Blázquez-M. $\Lambda$. Mezquiriz, op. cit., n" 24 .

34 H. Stern. "La mosaïque d"Orphée de Blanzy-les-Fismes (Aisne)". Gallia XIII (19:55): pp. 49 ss.

35 Sobre este mosaico y otros del Norte de Africa.: G. Guidi. "Orfeo, Liber Pater e Oceano in mosaici della Tripolitania". Afr. It. 6, 1935, pp. 110 ss.

36 J. M. Alvarez Martínez. "La iconografía de Orfeo en los mosaicos hispanorromanos" Mosaicos romanos. Rstudios sobre iconografia. Alberto Balil in memoriam. Guadalajara, 1990, pp. 34-40

37 R. Thouvenot. La maison d'Orphée à Volubilis. Publications du Service des Antiquités du Maroc, fasc. 6, 1941. pp. 43-47, fig. 1.

38 D. J. Smith. "Orpheus mosaics in Britain". Mosaïque. Recueil d'hommages á Hemi Stern. Paris, 1982, pp. 315 ss.

39 J. M. Alvarez Martíncz, op. cit., pp. 39-40.

40 M. de Vos. L'egittomamía in pitture e mosaici romano-campani della prima elà imperiale. Leiden, 1980.

41 I. Foucher. "Les mosaïques nilotiques africaines". Colloque la Mosaïque gréco-romaine I, pp. 137-145.

42 A. Blanco-J. M. Luzón. El mosaico de Neptumo en Itálica. Sevilla, 1974, pp. 41-46.

43 A. Blanco. Mosaicos romanos de Mérida, n" 9, láms. 12,18 y 19.

44 J. M. Alvarez Martínez, op. cit., pp. 41 y $46-4$ ?

45 A. Daviault-J. Lancha-L.A. López Palomo. lin mosaico con inscripciones. Publications de la Casa de Velázquez. Serie Etudes et Documents III. Madrid, 1987, pp. 17 ss. En este estudio se habla de las relaciones entre los asumios nilóticos y la comedia.Sobre este tema, verase: A. Daviault. "L'uxor Mastale: personnage de comédic latine". Hommages a E. Pascal. II. 1990, pp. 375 ss.

46 K. Schefuld. Die Wände Pompejis. Berlin, 1957. Para la clasificación de los mosaicos nilóticos africanos, resulıa útil el arículo de J. Lancha. "Deux fragments d'une mosä̈pue milotique conservés au Musée de Naples". MEFRA, 92, 1980, pp. 249-276.

47 A. Balil. "Mosaicos circenses de Barcelona y Gerona". BRA/H CLI, pp. 257-351.

48 K. M. D. Dunbabin, op. cil., pp. 89 ss. En el Mosaico de Cartago se ha querido ver una posible cabeza de serie de pavimentos de temas similares en las provineins occidentales del Imperio: M. Yacoub. "Le motif de cirque: un motif d'origine af ricaine?". Fith International Coloquium on Ancient Mosnics. Journal of Roman Archacolugy. Supplementary Series number nime.AnnArbor, 1994, pp. 149 ss.

49 K. M. D. Dunbabin. "The victorious Chariotecr on mosaics an related monuments". A.J.A. 86. 1, pf. 70-72. Igualunente, es interesante el trahajo de M. Ennaifer: "Le thème des chevaux vainqueurs á travers la serie des mosaïques africaines". MLFRA, 95, 1983, pp. 817 ss. Sobre los nombres de los cahallos, véase el reciente estudio de M. Darder Lisson. De nominibus equorum circensium. Pars Occidentis. Barcelona, 1996, passim.

50 Para las ejemplos emeritenses, bien significativos, véase: .f. Alvarez Sáenz de Buruaga. "Lna casa romana con valiosa pinturas, de Mérida". Habis-is (1974). pp. 173-174 y 178-79.; A. Blanco. Mosaicos romanos de Mérida, $\mathrm{n}^{\prime 4} 43$, pp. 5-46, láms. 76-79.; J. M. Alvarez Martínez, op. cil., pp. 81-82 y 86-89.

51 Los mosaicos africanos que reflejan aspectos de esa forma de vida son abundantes. Lina 
silllesis, ya superada, es la de. M. Tl. Preduenr-Canonge. La vie murve clans I'Afrigue romaine d'aprés les moscrïpues. Partis. s.a. Igualmente se encuentran referencias sobre estus asuntos en K. M. D. Dunbalin. op. cit., [1]. 109 ss.Observaciones interesantes ofr:: K. Weitzmam. "Représentations of Daily I ife". Age of Spiritualig: Late Antipue and lisuly

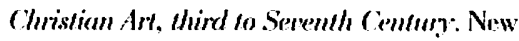
York. 1970, pp. 270 ss. Solore cste tema y la romparación de las escenas africauas ron las hispanas, véase: J. M. Blázquoz Martínez. "lll entomo cle las villas en los mosaicos de Afriea e lispania”. LAfrica momono. Nui del $X$ Conveguo di studio. Oristano. 11-1:3 dicember 190). Siassari. 1994. pp. 1.1?1-1.18?.

52 T. Sarnowski. Les representutions de rilles sur les mosaigues africaines terdires. Wroclaw. 1978

53 J. M. Blázquez. "El entorıo...", I'). 1.182-1.181.1.187.

54 .1. M. Blázyuez. "Arte y soriedaul on lus mosairos romanos de Navarra“ en Mosaicos romanos do España. Madrid, 1993, p. 59.

55 K. M. D. Dumbabin, op. ril., PJ. 109 ss.T. Sarnowski, op. cil. prtssim.

56 Siblyre el mausoleo tarraconense, víase: 11. Sibhlunk. Die Mosaikikupel ron (interolles. 2 Bule. Mainz. 1988. Especialmente. pp. 18-20

57 Siohre este mosairo, véase por ejemplo: D. Parrish. Season Mosaics of romom North 1frica. Roma, 1984, mosaico n" del cutálogo, pp. 111-113, láms. 15-16.

58 I. M. Nvarez Martinez-T'. Nugalesi Basarrate. "Los mosaicos de la rille rumuma de "Panes Perdidos" en Solama de los Barros (Barlajuz). Anas. 7-8 (en prensa).

59 A. Blanco. Mosaicos de Mérida, n" 39, p. +4, lámils. ?:3-74

60 I. M. Alvarez Martínez. Mosaicos de Mérila, ॥".3, pр. 37 ss.
61 J. M. Blázquez. "Mosaicos báquices de la Península lbérica". AEspA, vol. 57, 1" $149-150(1984)$.

62 M. Pellicer. "lixcavaciones en d yacimiento romano de los -Baños de la Reina”. Calpe: (Alicanto)" NAll. vill-lX (1966). pl. 172 ss. láms. $\mathrm{XXX}-\mathrm{XXXI}$. Muy parecida a las escenas del mosaico de Travesia de Pealro María Plano.

63 D. Fermández-Caliano. Comp/m/um /I. Moscarros li:Al.E, 138. Madrid. 1984, p. 171.

64 K. M. Dumbabin. op. cit., pp. 115 ss.

65 Sobre este importante mosaico. véase: 11. Stern. "l aes mosaïques de l'Eglise de Same Consiance à Rume". D.O.P. 12 (1950). 1. 190.: 15. B. Sear. Roman wall and raull mesarics Heiclelberg. 1977, n" 1+2, p. 1:31. líus. 5.5,4 y .56,1.

66 .I. M. Blazque\% el alii. "Iconogralïa de la vida cotidliana: cemas de caza" Mosaicos rommonos de Españo. Madrid, 199.3. pp. 24.5 ss.

67 J. M. Alvarez. Martínez. "La villa romana de "El I linujal" en la dehesa de "l.as 'liendas" (Mirida) N.A.II. -Arqueologin- I' 1976, IU. +51 is.

68 I. W. Sulomunson. La mosä̈une anx' cherand de SAntiquarium de Carthage. I a Ilıỵa. 1965).

69 J. . N. Nuarez Martínez. Mosaicos de Mérida. "1" 1+. p). 82-83

70 I. M. Alvarez Martinez-T. Nogales Basurrace. "Algunas consideraciones sobre la donaracion de sillae del territorium emerilense: musivaria y esculıura". Sindirt ISistorica. Historia antigua. rol. X-XI (1992-199:3), pp. 277-278.

71 J. Aymard. "Quelques scénes de chasse: sur une mosä̈que de l'Aniquarium". Mel de:Arch. ef dWist. I.IV (1937), pl. t4 ss.

72 1. lavin. "The hunting mosaics of $A$ miod, and ilueir sourees". D.O.P., 17. 
73 P. Romanelli. "Riflessi di vina locale nei mosaici africani". CMOR I, p. 285, fig. 9

74 R. Bianchi Bandinelli. Rome. El fin del arte antiguo. Madrid, 1971, p. 310, lig. 289.

75 G. Vinicio Gentili. La villa imperiale di Piazza Armerina. Roma, 1971, p. 31, fig. 14. Más reciente: $A$. Carandini- $A$. Ricci- M. de Vos. Filosofann. The villa of Piazza Armerina. Palermo, 1982, pp. 176 ss.. fig. 100

76 J. M. Blázquez-M. A. Mezquiriz. op. cit. pp. 64 ss.

77 Blázquez--Mezquiriz, art. cit. PP. 64 ss.
78 D. Pernández-Galiano. "Influenciats oricutales en la musivaria hispánica” . III Colormio Internazionale sul Mosaico antico. Ravenua. 1984, pp. 418-20; (. I.ópez Montengudo. "La caza en el mosaico romumo. lconografía y simbolismo". Arte. sociedad, economia y religión durante el Bajo Imperio y la antigïedad Tardía. Antig. crist. III, 1991, p. 498.; J. M. Alvarez Marıíız-'T. Nogales, arı. cit. pp. 278 y 286.

79 A. Carandini. "Ricerche sui problemi dellultima pittura tardo-antica nel bacino del Mediterraneo meridionale”. Archeologia Classica, vol. XIV. fasc. $2(1962)$, p. 234. 